\title{
Supported Materials
}

\section{Surface ozone impacts on major crop production in China from 2010 to 2017}

Dianyi Li, Drew Shindell, Dian Ding, Xiao Lu, Lin Zhang, Yuqiang Zhang

Table S1. Evaluation results of simulated AOT40 and observational Data during growth season (April to September) from 2013 to 2017.

\begin{tabular}{|l|r|r|r|r|r|r|}
\hline Year & $\begin{array}{l}\text { modeled mean } \\
(\mathrm{ppm} \mathrm{h})\end{array}$ & $\begin{array}{l}\text { observation } \\
\text { mean }(\mathrm{ppm} \mathrm{h})\end{array}$ & $\begin{array}{l}\text { Mean Bias } \\
(\mathrm{ppm} \mathrm{h})\end{array}$ & $\begin{array}{l}\text { Normalized } \\
\text { Mean Bias }\end{array}$ & $\begin{array}{l}\text { Mean } \\
\text { Error }\end{array}$ & $\begin{array}{l}\text { Normalized } \\
\text { Mean Error }\end{array}$ \\
\hline 2013 & 21200.08 & 23362.38 & -2162.30 & $-9 \%$ & 9979.04 & $43 \%$ \\
\hline 2014 & 22591.57 & 24196.98 & -1605.42 & $-7 \%$ & 11178.88 & $46 \%$ \\
\hline 2015 & 22398.99 & 23459.15 & -1060.16 & $-5 \%$ & 10609.44 & $45 \%$ \\
\hline 2016 & 20851.19 & 23579.00 & -2727.81 & $-12 \%$ & 9998.78 & $42 \%$ \\
\hline 2017 & 16796.01 & 23313.05 & -6517.04 & $-28 \%$ & 10973.21 & $47 \%$ \\
\hline
\end{tabular}

Table S2. 8-year average annual accumulated AOT40 in China provinces. The provinces are listed following the orders of their magnitudes. Unit of $\mathbf{p p m} \mathbf{h}$.

\begin{tabular}{|c|c|c|c|c|c|}
\hline & Provinces & AOT40 (ppm h) & & Provinces & AOT40 (ppm h) \\
\hline 1 & Xizang & 41.47 & 18 & Hubei & 27.95 \\
\hline 2 & Tianjin & 34.79 & 19 & Chongqing & 26.79 \\
\hline 3 & Qinghai & 34.51 & 20 & Shanghai & 26.38 \\
\hline 4 & Shanxi & 33.65 & 21 & Xinjiang Uygur & 25.01 \\
\hline 5 & Hebei & 32.64 & 22 & Jiangxi & 24.29 \\
\hline 6 & Shaanxi & 32.30 & 23 & Nei Mongol & 24.03 \\
\hline 7 & Henan & 32.25 & 24 & Fujian & 23.96 \\
\hline 8 & Liaoning & 31.72 & 25 & Yunnan & 23.35 \\
\hline 9 & Beijing & 31.68 & 26 & Hunan & 22.72 \\
\hline 10 & Sichuan & 30.93 & 27 & Taiwan & 22.49 \\
\hline 11 & Anhui & 30.81 & 28 & Heilongjiang & 22.48 \\
\hline 12 & Shandong & 30.61 & 29 & Guizhou & 21.34 \\
\hline 13 & Ningxia Hui & 29.76 & 30 & Guangdong & 19.85 \\
\hline 14 & Jiangsu & 29.58 & 31 & Hainan & 18.08 \\
\hline 15 & Gansu & 29.10 & 32 & Guangxi & 17.81 \\
\hline 16 & Jilin & 28.93 & 33 & HongKong & NA \\
\hline 17 & Zhejiang & 28.31 & 34 & Macao & NA \\
\hline
\end{tabular}


Table S3. 8-year average of accumulated AOT40 and RYL for wheat during growing season (March, April, and May).

\begin{tabular}{|c|c|c|c|c|c|c|c|}
\hline & Provinces & $\begin{array}{l}\text { Growing } \\
\text { season AOT40 } \\
(\mathrm{ppm} \text { h) }\end{array}$ & RYL & & Provinces & $\begin{array}{l}\text { Growing } \\
\text { season AOT40 } \\
(\mathrm{ppm} \text { h) }\end{array}$ & RYL \\
\hline 1 & Xizang & 14.99 & $25.14 \%$ & 18 & Jilin & 9.33 & $16.02 \%$ \\
\hline 2 & Yunnan & 12.60 & $21.29 \%$ & 19 & Liaoning & 8.89 & $15.31 \%$ \\
\hline 3 & Qinghai & 11.77 & $19.95 \%$ & 20 & Hebei & 8.68 & $14.97 \%$ \\
\hline 4 & Sichuan & 11.64 & $19.74 \%$ & 21 & Nei Mongol & 8.54 & $14.76 \%$ \\
\hline 5 & Anhui & 10.89 & $18.53 \%$ & 22 & Fujian & 8.49 & $14.67 \%$ \\
\hline 6 & Zhejiang & 10.60 & $18.06 \%$ & 23 & Heilongjiang & 8.44 & $14.59 \%$ \\
\hline 7 & Henan & 10.52 & $17.95 \%$ & 24 & Jiangxi & 8.37 & $14.47 \%$ \\
\hline 8 & Shaanxi & 10.47 & $17.85 \%$ & 25 & Beijing & 8.29 & $14.34 \%$ \\
\hline 9 & Jiangsu & 10.34 & $17.64 \%$ & 26 & Tianjin & 8.12 & $14.08 \%$ \\
\hline 10 & Hubei & 10.02 & $17.13 \%$ & 27 & Taiwan & 7.99 & $13.86 \%$ \\
\hline 11 & Shanxi & 9.74 & $16.69 \%$ & 28 & Xinjiang Uygur & 7.98 & $13.85 \%$ \\
\hline 12 & Shandong & 9.66 & $16.56 \%$ & 29 & Hunan & 7.92 & $13.74 \%$ \\
\hline 13 & Gansu & 9.64 & $16.52 \%$ & 30 & Guangxi & 6.83 & $11.99 \%$ \\
\hline 14 & Ningxia Hui & 9.63 & $16.51 \%$ & 31 & Guangdong & 5.91 & $10.52 \%$ \\
\hline 15 & Shanghai & 9.63 & $16.50 \%$ & 32 & Hainan & 4.43 & $8.13 \%$ \\
\hline 16 & Chongqing & 9.62 & $16.49 \%$ & 33 & HongKong & NA & NA \\
\hline 17 & Guizhou & 9.47 & $16.24 \%$ & 34 & Macao & NA & NA \\
\hline
\end{tabular}

Table S4. As for Table S3 but for double rice (growing season May, June, July for early rice, and September, October and November for late rice).

\begin{tabular}{|l|l|r|r|}
\hline & Provinces & $\begin{array}{l}\text { Growing } \\
\text { Season AOT40 } \\
\text { (ppm h) }\end{array}$ & $\begin{array}{l}\text { Double } \\
\text { Early } \\
\text { Rice }\end{array}$ \\
\hline 1 & Anhui & 12.09 & $10.71 \%$ \\
\hline 2 & Hubei & 10.23 & $9.99 \%$ \\
\hline 3 & Zhejiang & 8.72 & $9.40 \%$ \\
\hline 4 & Hunan & 6.57 & $8.56 \%$ \\
\hline 5 & Jiangxi & 6.41 & $8.50 \%$ \\
\hline 6 & Fujian & 4.46 & $7.74 \%$ \\
\hline 7 & Yunnan & 3.52 & $7.37 \%$ \\
\hline 8 & Guangxi & 3.41 & $7.33 \%$ \\
\hline 9 & Guangdong & 2.78 & $7.08 \%$ \\
\hline 10 & Taiwan & 2.43 & $6.95 \%$ \\
\hline 11 & Hainan & 1.84 & $6.72 \%$ \\
\hline 12 & HongKong & NA & NA \\
\hline 13 & Macao & NA & NA \\
\hline
\end{tabular}

\begin{tabular}{|l|l|r|r|} 
& Provinces & $\begin{array}{l}\text { Growing } \\
\text { Season AOT40 } \\
\text { (ppm h) }\end{array}$ & $\begin{array}{l}\text { Double } \\
\text { Late } \\
\text { Rice }\end{array}$ \\
\hline 1 & Fujian & 6.47 & $8.53 \%$ \\
\hline 2 & Jiangxi & 6.39 & $8.49 \%$ \\
\hline 3 & Guangdong & 6.35 & $8.48 \%$ \\
\hline 4 & Anhui & 6.17 & $8.41 \%$ \\
\hline 5 & Hubei & 5.99 & $8.34 \%$ \\
\hline 6 & Zhejiang & 5.97 & $8.33 \%$ \\
\hline 7 & Hunan & 5.85 & $8.28 \%$ \\
\hline 8 & Taiwan & 5.62 & $8.19 \%$ \\
\hline 9 & Hainan & 5.27 & $8.06 \%$ \\
\hline 10 & Guangxi & 4.66 & $7.82 \%$ \\
\hline 11 & Yunnan & 2.86 & $7.11 \%$ \\
\hline 12 & HongKong & NA & NA \\
\hline 13 & Macao & NA & NA \\
\hline
\end{tabular}


Table S5. As for Table S3 but for single rice (growing season August, September, and October).

\begin{tabular}{|c|c|c|c|c|c|c|c|}
\hline & Provinces & $\begin{array}{l}\text { Growing } \\
\text { Season AOT40 } \\
(\text { ppm h) }\end{array}$ & RYL & & Provinces & $\begin{array}{l}\text { Growing } \\
\text { Season AOT40 } \\
(\text { ppm h) }\end{array}$ & RYL \\
\hline 1 & Tianjin & 10.81 & $10.22 \%$ & 18 & Chongqing & 7.24 & $8.82 \%$ \\
\hline 2 & Shanxi & 9.76 & $9.81 \%$ & 19 & Jilin & 6.92 & $8.70 \%$ \\
\hline 3 & Henan & 9.41 & $9.67 \%$ & 20 & Shanghai & 6.81 & $8.66 \%$ \\
\hline 4 & Shaanxi & 9.08 & $9.54 \%$ & 21 & Hunan & 6.67 & $8.60 \%$ \\
\hline 5 & Hebei & 9.06 & $9.53 \%$ & 22 & Gansu & 6.45 & $8.52 \%$ \\
\hline 6 & Shandong & 8.84 & $9.45 \%$ & 23 & Fujian & 6.30 & $8.46 \%$ \\
\hline 7 & Anhui & 8.83 & $9.44 \%$ & 24 & Sichuan & 5.46 & $8.13 \%$ \\
\hline 8 & Liaoning & 8.82 & $9.44 \%$ & 25 & Xinjiang Uygur & 5.04 & $7.97 \%$ \\
\hline 9 & Beijing & 8.47 & $9.30 \%$ & 26 & Nei Mongol & 4.46 & $7.74 \%$ \\
\hline 10 & Jiangsu & 8.18 & $9.19 \%$ & 27 & Heilongjiang & 4.18 & $7.63 \%$ \\
\hline 11 & Ningxia Hui & 8.00 & $9.12 \%$ & 28 & Guizhou & 4.18 & $7.63 \%$ \\
\hline 12 & Hubei & 7.99 & $9.12 \%$ & 29 & Guangxi & 4.14 & $7.62 \%$ \\
\hline 13 & Zhejiang & 7.30 & $8.85 \%$ & 30 & Yunnan & 2.02 & $6.79 \%$ \\
\hline 14 & Jiangxi & 7.28 & $8.84 \%$ & & & & \\
\hline
\end{tabular}

Table S6. As for Table S3 but for Maize (growing season June, July and August for north maize, and August, September and October for south maize).

\begin{tabular}{|c|c|c|c|c|c|c|c|}
\hline & Provinces & $\begin{array}{l}\text { Growing } \\
\text { Season AOT40 } \\
(\mathrm{ppm} h)\end{array}$ & $\begin{array}{l}\text { North } \\
\text { Maize }\end{array}$ & & Provinces & $\begin{array}{l}\text { Growing } \\
\text { Season AOT40 } \\
(\text { ppm h) }\end{array}$ & $\begin{array}{l}\text { South } \\
\text { Maize }\end{array}$ \\
\hline 1 & Tianjin & 20.24 & $5.29 \%$ & 1 & Jiangsu & 8.18 & $0.94 \%$ \\
\hline 2 & Beijing & 17.92 & $4.45 \%$ & 2 & Hubei & 7.99 & $0.89 \%$ \\
\hline 3 & Hebei & 17.80 & $4.41 \%$ & 3 & Zhejiang & 7.30 & $0.64 \%$ \\
\hline 4 & Shanxi & 16.49 & $3.94 \%$ & 4 & Jiangxi & 7.28 & $0.64 \%$ \\
\hline 5 & Liaoning & 15.57 & $3.61 \%$ & 5 & Chongqing & 7.24 & $0.65 \%$ \\
\hline 6 & Shandong & 13.93 & $3.01 \%$ & 6 & Shanghai & 6.81 & $0.50 \%$ \\
\hline 7 & Henan & 13.71 & $2.93 \%$ & 7 & Xizang & 6.81 & $0.50 \%$ \\
\hline 8 & Jilin & 12.88 & $2.64 \%$ & 8 & Hunan & 6.67 & $0.47 \%$ \\
\hline 9 & Shaanxi & 12.70 & $2.57 \%$ & 9 & Fujian & 6.30 & $0.37 \%$ \\
\hline 10 & Ningxia Hui & 11.02 & $1.97 \%$ & 10 & Guangdong & 5.48 & $0.20 \%$ \\
\hline 11 & Anhui & 10.77 & $1.88 \%$ & 11 & Sichuan & 5.46 & $0.18 \%$ \\
\hline 12 & Heilongjiang & 9.08 & $1.27 \%$ & 12 & Taiwan & 5.02 & $0.11 \%$ \\
\hline 13 & Gansu & 8.95 & $1.22 \%$ & 13 & Guizhou & 4.18 & $0.00 \%$ \\
\hline 14 & Nei Mongol & 8.69 & $1.13 \%$ & 14 & Guangxi & 4.14 & $0.00 \%$ \\
\hline 15 & Xinjiang Uygur & 7.57 & $0.74 \%$ & 15 & Hainan & 3.85 & $0.00 \%$ \\
\hline 16 & Qinghai & 6.58 & $0.48 \%$ & 16 & Yunnan & 2.02 & $0.00 \%$ \\
\hline
\end{tabular}


Table S7. As for Table S3 but for soybean (growing season June, July and August).

\begin{tabular}{|c|c|c|c|c|c|c|c|}
\hline & Provinces & $\begin{array}{l}\text { Growing } \\
\text { Season AOT40 } \\
(\mathrm{ppm} \text { h) }\end{array}$ & RYL & & Provinces & $\begin{array}{l}\text { Growing } \\
\text { Season AOT40 } \\
(p p m \text { h) }\end{array}$ & RYL \\
\hline 1 & Tianjin & 20.24 & $11.48 \%$ & 18 & Chongqing & 8.40 & $0.00 \%$ \\
\hline 2 & Beijing & 17.92 & $8.79 \%$ & 19 & Xinjiang Uygur & 7.57 & $0.00 \%$ \\
\hline 3 & Hebei & 17.80 & $8.65 \%$ & 20 & Zhejiang & 7.55 & $0.00 \%$ \\
\hline 4 & Shanxi & 16.49 & $7.13 \%$ & 21 & Qinghai & 6.58 & $0.00 \%$ \\
\hline 5 & Liaoning & 15.57 & $6.07 \%$ & 22 & Sichuan & 6.15 & $0.00 \%$ \\
\hline 6 & Shandong & 13.93 & $4.15 \%$ & 23 & Xizang & 6.02 & $0.00 \%$ \\
\hline 7 & Henan & 13.71 & $3.90 \%$ & 24 & Jiangxi & 5.59 & $0.00 \%$ \\
\hline 8 & Jilin & 12.9 & $3.02 \%$ & 25 & Hunan & 5.49 & $0.00 \%$ \\
\hline 9 & Shaanxi & 12.88 & $2.73 \%$ & 26 & Fujian & 3.62 & $0.00 \%$ \\
\hline 10 & Jiangsu & 12.70 & $1.49 \%$ & 27 & Guizhou & 3.57 & $0.00 \%$ \\
\hline 11 & Ningxia Hui & 11.02 & $1.24 \%$ & 28 & Guangdong & 2.42 & $0.00 \%$ \\
\hline 12 & Anhui & 10.77 & $0.70 \%$ & 29 & Guangxi & 2.36 & $0.00 \%$ \\
\hline 13 & Shanghai & 9.28 & $0.47 \%$ & 30 & Taiwan & 2.17 & $0.00 \%$ \\
\hline 14 & Heilongjiang & 9.08 & $0.00 \%$ & 31 & Hainan & 1.54 & $0.00 \%$ \\
\hline 15 & Gansu & 8.95 & $0.31 \%$ & 32 & Yunnan & 1.39 & $0.00 \%$ \\
\hline 16 & Hubei & 8.78 & $0.01 \%$ & 33 & HongKong & NA & NA \\
\hline 17 & Nei Mongol & 8.69 & $0.00 \%$ & 34 & Macao & $\mathrm{NA}$ & $\mathrm{NA}$ \\
\hline
\end{tabular}


Table S8. Province level of ozone induced production loss for wheat from 2010 to 2017 (1000 metric tons).

\begin{tabular}{|c|c|c|c|c|c|c|c|c|c|}
\hline Province & 2010 & 2011 & 2012 & 2013 & 2014 & 2015 & 2016 & 2017 & $\begin{array}{l}\text { 2010-2017 } \\
\text { Average }\end{array}$ \\
\hline Henan & 2636 & 3015 & 3882 & 5514 & 7046 & 7273 & 7270 & 5240 & 5234 \\
\hline Shandong & 2228 & 2469 & 3757 & 4918 & 6404 & 6829 & 6690 & 4831 & 4766 \\
\hline Hebei & 1237 & 1052 & 2097 & 2784 & 3865 & 4424 & 4044 & 2805 & 2788 \\
\hline Jiangsu & 1389 & 2067 & 2114 & 2995 & 3532 & 3547 & 3060 & 2564 & 2658 \\
\hline Anhui & 1457 & 1978 & 2120 & 2816 & 3444 & 3325 & 3055 & 2468 & 2583 \\
\hline Shaanxi & 820 & 863 & 1090 & 1718 & 2037 & 2100 & 2127 & 1444 & 1525 \\
\hline Sichuan & 785 & 925 & 1011 & 1243 & 1218 & 1260 & 1218 & 874 & 1067 \\
\hline Hubei & 556 & 794 & 749 & 1107 & 1254 & 1226 & 1147 & 922 & 970 \\
\hline Shanxi & 427 & 405 & 659 & 996 & 1318 & 1423 & 1427 & 966 & 953 \\
\hline Gansu & 466 & 525 & 678 & 1064 & 1195 & 1101 & 1086 & 748 & 858 \\
\hline $\begin{array}{r}\text { Xinjiang } \\
\text { Uygur }\end{array}$ & 415 & 541 & 663 & 839 & 1087 & 938 & 780 & 653 & 740 \\
\hline Yunnan & 412 & 354 & 528 & 566 & 602 & 546 & 596 & 452 & 507 \\
\hline Nei Mongol & 187 & 181 & 241 & 554 & 614 & 641 & 554 & 374 & 418 \\
\hline Tianjin & 105 & 80 & 171 & 240 & 303 & 407 & 373 & 266 & 243 \\
\hline Chongqing & 153 & 193 & 184 & 263 & 261 & 272 & 257 & 182 & 221 \\
\hline Beijing & 67 & 51 & 101 & 165 & 221 & 277 & 246 & 164 & 161 \\
\hline Guizhou & 134 & 131 & 162 & 220 & 218 & 206 & 200 & 140 & 176 \\
\hline Ningxia Hui & 82 & 89 & 114 & 205 & 231 & 212 & 206 & 140 & 160 \\
\hline Heilongjiang & 58 & 50 & 53 & 130 & 146 & 151 & 132 & 103 & 103 \\
\hline Hunan & 31 & 44 & 37 & 56 & 60 & 59 & 55 & 44 & 48 \\
\hline Qinghai & 26 & 31 & 39 & 60 & 69 & 63 & 62 & 41 & 49 \\
\hline Xizang & 65 & 70 & 90 & 75 & 73 & 79 & 84 & 58 & 74 \\
\hline Zhejiang & 31 & 44 & 41 & 53 & 60 & 60 & 46 & 43 & 47 \\
\hline Liaoning & 10 & 10 & 14 & 24 & 28 & 33 & 28 & 21 & 21 \\
\hline Jiangxi & 14 & 21 & 17 & 23 & 26 & 25 & 21 & 19 & 21 \\
\hline Shanghai & 8 & 13 & 12 & 17 & 19 & 19 & 15 & 14 & 15 \\
\hline Fujian & 5 & 7 & 5 & 7 & 7 & 7 & 7 & 6 & 6 \\
\hline Guangxi & 5 & 4 & 6 & 7 & 7 & 7 & 8 & 5 & 6 \\
\hline Jilin & 2 & 2 & 3 & 5 & 6 & 7 & 6 & 4 & 4 \\
\hline Guangdong & 0 & 0 & 0 & 0 & 0 & 0 & 0 & 0 & 0 \\
\hline Hainan & 0 & 0 & 0 & 0 & 0 & 0 & 0 & 0 & 0 \\
\hline HongKong & 0 & 0 & 0 & 0 & 0 & 0 & 0 & 0 & 0 \\
\hline Macao & 0 & 0 & 0 & 0 & 0 & 0 & 0 & 0 & 0 \\
\hline Taiwan & 0 & 0 & 0 & 0 & 0 & 0 & 0 & 0 & 0 \\
\hline China & 13811 & 16009 & 20638 & 28664 & 35351 & 36517 & 34800 & 25591 & 26422 \\
\hline
\end{tabular}


Table S9. As Table S8 but for double early rice (1000 Mt)

\begin{tabular}{|r|r|r|r|r|r|r|r|r|r|}
\hline Province & $\mathbf{2 0 1 0}$ & $\mathbf{2 0 1 1}$ & $\mathbf{2 0 1 2}$ & $\mathbf{2 0 1 3}$ & $\mathbf{2 0 1 4}$ & $\mathbf{2 0 1 5}$ & $\mathbf{2 0 1 6}$ & $\mathbf{2 0 1 7}$ & $\begin{array}{r}\mathbf{2 0 1 0 - 2 0 1 7} \\
\text { Average }\end{array}$ \\
\hline Hunan & 799 & 880 & 856 & 875 & 1002 & 937 & 917 & 841 & 888 \\
\hline Jiangxi & 883 & 946 & 884 & 835 & 942 & 849 & 821 & 775 & 867 \\
\hline Guangdong & 443 & 486 & 451 & 462 & 496 & 469 & 469 & 464 & 467 \\
\hline Guangxi & 420 & 459 & 429 & 453 & 479 & 472 & 449 & 432 & 449 \\
\hline Fujian & 214 & 228 & 218 & 214 & 234 & 217 & 221 & 211 & 220 \\
\hline Anhui & 288 & 303 & 318 & 287 & 296 & 245 & 228 & 208 & 272 \\
\hline Hubei & 242 & 203 & 210 & 191 & 220 & 202 & 145 & 121 & 192 \\
\hline Zhejiang & 141 & 146 & 135 & 136 & 146 & 146 & 155 & 142 & 143 \\
\hline Taiwan & 52 & 52 & 53 & 52 & 55 & 54 & 56 & 54 & 53 \\
\hline Hainan & 34 & 39 & 36 & 38 & 39 & 40 & 39 & 37 & 38 \\
\hline Yunnan & 9 & 9 & 10 & 9 & 10 & 11 & 10 & 10 & 10 \\
\hline HongKong & 0 & 0 & 0 & 0 & 0 & 0 & 0 & 0 & 0 \\
\hline Macao & 0 & 0 & 0 & 0 & 0 & 0 & 0 & 0 & 0 \\
\hline Total & $\mathbf{3 5 2 5}$ & $\mathbf{3 7 5 1}$ & $\mathbf{3 6 0 0}$ & $\mathbf{3 5 5 2}$ & $\mathbf{3 9 1 9}$ & $\mathbf{3 6 4 2}$ & $\mathbf{3 5 1 0}$ & $\mathbf{3 2 9 5}$ & $\mathbf{3 5 9 9}$ \\
\hline
\end{tabular}

Table S10. As Table S8 but for double late rice (1000 Mt)

\begin{tabular}{|r|r|r|r|r|r|r|r|r|r|}
\hline Province & $\mathbf{2 0 1 0}$ & $\mathbf{2 0 1 1}$ & $\mathbf{2 0 1 2}$ & $\mathbf{2 0 1 3}$ & $\mathbf{2 0 1 4}$ & $\mathbf{2 0 1 5}$ & $\mathbf{2 0 1 6}$ & $\mathbf{2 0 1 7}$ & $\begin{array}{r}\text { 2010 -2017 } \\
\text { Average }\end{array}$ \\
\hline Jiangxi & 820 & 865 & 919 & 910 & 915 & 872 & 747 & 750 & 850 \\
\hline Hunan & 745 & 807 & 858 & 928 & 923 & 918 & 812 & 775 & 846 \\
\hline Guangdong & 515 & 557 & 609 & 607 & 615 & 584 & 534 & 523 & 568 \\
\hline Guangxi & 451 & 492 & 516 & 531 & 508 & 490 & 480 & 444 & 489 \\
\hline Fujian & 220 & 233 & 264 & 264 & 280 & 260 & 225 & 226 & 247 \\
\hline Anhui & 217 & 219 & 230 & 241 & 233 & 201 & 171 & 158 & 209 \\
\hline Hubei & 194 & 156 & 169 & 175 & 181 & 175 & 118 & 100 & 158 \\
\hline Zhejiang & 109 & 117 & 120 & 126 & 138 & 130 & 120 & 122 & 123 \\
\hline Taiwan & 53 & 58 & 62 & 70 & 79 & 68 & 62 & 62 & 64 \\
\hline Hainan & 41 & 44 & 47 & 49 & 48 & 46 & 44 & 42 & 45 \\
\hline Yunnan & 9 & 9 & 9 & 9 & 10 & 10 & 10 & 10 & 9 \\
\hline HongKong & 0 & 0 & 0 & 0 & 0 & 0 & 0 & 0 & 0 \\
\hline Macao & 0 & 0 & 0 & 0 & 0 & 0 & 0 & 0 & 0 \\
\hline Total & $\mathbf{3 3 7 4}$ & $\mathbf{3 5 5 7}$ & $\mathbf{3 8 0 3}$ & $\mathbf{3 9 1 0}$ & $\mathbf{3 9 3 0}$ & $\mathbf{3 7 5 4}$ & $\mathbf{3 3 2 3}$ & $\mathbf{3 2 1 2}$ & $\mathbf{3 6 0 8}$ \\
\hline
\end{tabular}


Table S 11. As for Table S8 but for single rice (1000 Mt).

\begin{tabular}{|c|c|c|c|c|c|c|c|c|c|}
\hline Province & 2010 & 2011 & 2012 & 2013 & 2014 & 2015 & 2016 & 2017 & $\begin{array}{r}\text { 2010-2017 } \\
\text { Average } \\
\end{array}$ \\
\hline Anhui & 1692 & 1746 & 1840 & 1936 & 2043 & 2142 & 1932 & 1918 & 1906 \\
\hline Jiangsu & 1551 & 1631 & 1700 & 1825 & 1899 & 1907 & 1739 & 1716 & 1746 \\
\hline Hubei & 1128 & 1294 & 1323 & 1419 & 1452 & 1518 & 1437 & 1411 & 1373 \\
\hline Sichuan & 1048 & 1071 & 1080 & 1245 & 1209 & 1258 & 1219 & 1091 & 1152 \\
\hline Hunan & 767 & 815 & 830 & 799 & 830 & 829 & 763 & 800 & 804 \\
\hline Zhejiang & 656 & 723 & 769 & 790 & 836 & 799 & 694 & 730 & 750 \\
\hline Henan & 420 & 433 & 458 & 492 & 501 & 526 & 482 & 456 & 471 \\
\hline Jiangxi & 303 & 357 & 418 & 450 & 506 & 516 & 519 & 542 & 451 \\
\hline Chongqing & 382 & 391 & 388 & 451 & 442 & 456 & 439 & 399 & 418 \\
\hline Fujian & 269 & 288 & 327 & 331 & 359 & 335 & 299 & 321 & 316 \\
\hline Guizhou & 257 & 265 & 276 & 308 & 301 & 309 & 289 & 272 & 285 \\
\hline Liaoning & 250 & 252 & 264 & 282 & 299 & 296 & 294 & 285 & 278 \\
\hline Heilongjiang & 256 & 252 & 259 & 279 & 283 & 294 & 283 & 269 & 272 \\
\hline Yunnan & 248 & 261 & 268 & 285 & 286 & 287 & 275 & 264 & 272 \\
\hline Jilin & 182 & 186 & 190 & 205 & 213 & 218 & 208 & 197 & 200 \\
\hline Shandong & 145 & 150 & 159 & 171 & 178 & 180 & 176 & 169 & 166 \\
\hline Shanghai & 87 & 95 & 99 & 105 & 111 & 110 & 98 & 99 & 101 \\
\hline Shaanxi & 86 & 87 & 91 & 110 & 105 & 110 & 105 & 95 & 99 \\
\hline Hebei & 84 & 86 & 86 & 95 & 99 & 104 & 106 & 102 & 95 \\
\hline Guangxi & 70 & 80 & 85 & 93 & 93 & 90 & 89 & 93 & 87 \\
\hline $\begin{array}{r}\text { Xinjiang } \\
\text { Uygur }\end{array}$ & 35 & 37 & 38 & 40 & 41 & 39 & 41 & 39 & 39 \\
\hline Tianjin & 25 & 26 & 26 & 28 & 29 & 32 & 32 & 31 & 29 \\
\hline Nei Mongol & 23 & 24 & 24 & 27 & 28 & 28 & 27 & 26 & 26 \\
\hline Ningxia Hui & 16 & 17 & 17 & 20 & 20 & 20 & 20 & 19 & 19 \\
\hline Beijing & 4 & 5 & 5 & 5 & 5 & 5 & 5 & 5 & 5 \\
\hline Shanxi & 4 & 4 & 5 & 5 & 5 & 6 & 6 & 5 & 5 \\
\hline Gansu & 4 & 4 & 5 & 6 & 5 & 5 & 5 & 5 & 5 \\
\hline Total & 9992 & 10580 & 11030 & 11802 & 12178 & 12419 & 11582 & 11359 & 11370 \\
\hline
\end{tabular}


Table S12. As for Table S8 but for north and south maize (1000 Mt)

\begin{tabular}{|c|c|c|c|c|c|c|c|c|c|}
\hline \multicolumn{10}{|c|}{ North maize } \\
\hline Province & 2010 & 2011 & 2012 & 2013 & 2014 & 2015 & 2016 & 2017 & $\begin{array}{r}2010-2017 \\
\text { Average } \\
\end{array}$ \\
\hline Hebei & 1243 & 1200 & 1019 & 1186 & 1609 & 1806 & 1580 & 1202 & 1356 \\
\hline Shandong & 898 & 992 & 968 & 1068 & 1091 & 1305 & 1134 & 808 & 1033 \\
\hline Henan & 550 & 767 & 739 & 607 & 851 & 850 & 789 & 526 & 710 \\
\hline Jilin & 515 & 509 & 500 & 613 & 821 & 865 & 858 & 420 & 638 \\
\hline Liaoning & 438 & 455 & 427 & 517 & 681 & 729 & 702 & 427 & 547 \\
\hline Heilongjiang & 383 & 392 & 420 & 420 & 469 & 570 & 563 & 243 & 432 \\
\hline Shanxi & 207 & 261 & 216 & 238 & 307 & 330 & 279 & 216 & 257 \\
\hline Shaanxi & 113 & 214 & 157 & 210 & 359 & 359 & 278 & 189 & 235 \\
\hline Nei Mongol & 167 & 175 & 149 & 164 & 206 & 217 & 212 & 113 & 175 \\
\hline Tianjin & 130 & 114 & 106 & 136 & 193 & 229 & 193 & 149 & 156 \\
\hline Anhui & 110 & 126 & 152 & 130 & 130 & 153 & 159 & 105 & 133 \\
\hline Beijing & 106 & 99 & 75 & 82 & 121 & 139 & 125 & 97 & 106 \\
\hline $\begin{array}{r}\text { Xinjiang } \\
\text { Uygur }\end{array}$ & 12 & 44 & 51 & 89 & 98 & 92 & 57 & 25 & 58 \\
\hline Gansu & 9 & 23 & 11 & 27 & 47 & 48 & 31 & 21 & 27 \\
\hline Ningxia Hui & 7 & 10 & 7 & 12 & 14 & 14 & 11 & 8 & 10 \\
\hline Qinghai & 0 & 0 & 0 & 0 & 0 & 0 & 0 & 0 & 0 \\
\hline \multicolumn{10}{|c|}{ South Maize } \\
\hline Sichuan & 48 & 98 & 81 & 203 & 146 & 210 & 168 & 9.16 & 121 \\
\hline Jiangsu & 33 & 26 & 32 & 85 & 53 & 82 & 44 & 27 & 48 \\
\hline Chongqing & 9 & 22 & 27 & 73 & 58 & 86 & 57 & 2 & 42 \\
\hline Hubei & 6 & 12 & 26 & 39 & 36 & 56 & 31 & 1 & 26 \\
\hline Hunan & 0 & 1 & 6 & 7 & 7 & 11 & 4 & 0 & 4 \\
\hline Xizang & 0 & 0 & 1 & 5 & 6 & 7 & 6 & 5 & 4 \\
\hline Guizhou & 0 & 0 & 0 & 4 & 1 & 6 & 1 & 0 & 1 \\
\hline Yunnan & 0 & 0 & 0 & 1 & 0 & 0 & 0 & 0 & 0 \\
\hline Guangxi & 0 & 0 & 1 & 0 & 0 & 0 & 0 & 0 & 0 \\
\hline Taiwan & 0 & 0 & 0 & 1 & 1 & 1 & 0 & 0 & 0 \\
\hline Guangdong & 0 & 0 & 0 & 0 & 0 & 0 & 0 & 0 & 0 \\
\hline Zhejiang & 0 & 0 & 0 & 1 & 1 & 1 & 0 & 0 & 0 \\
\hline Jiangxi & 0 & 0 & 0 & 0 & 0 & 0 & 0 & 0 & 0 \\
\hline Fujian & 0 & 0 & 0 & 0 & 0 & 0 & 0 & 0 & 0 \\
\hline Shanghai & 0 & 0 & 0 & 0 & 0 & 0 & 0 & 0 & 0 \\
\hline Hainan & 0 & 0 & 0 & 0 & 0 & 0 & 0 & 0 & 0 \\
\hline HongKong & 0 & 0 & 0 & 0 & 0 & 0 & 0 & 0 & 0 \\
\hline Macao & 0 & 0 & 0 & 0 & 0 & 0 & 0 & 0 & 0 \\
\hline China & 4984 & 5540 & 5171 & 5918 & 7306 & 8166 & 7282 & 4593 & 6119 \\
\hline
\end{tabular}


Table S13. As for Table S8 but for soybean (1000 Mt).

\begin{tabular}{|c|c|c|c|c|c|c|c|c|c|}
\hline Province & 2010 & 2011 & 2012 & 2013 & 2014 & 2015 & 2016 & 2017 & $\begin{array}{r}2010-2017 \\
\text { Average } \\
\end{array}$ \\
\hline Heilongjiang & 103 & 84 & 84 & 76 & 96 & 83 & 93 & 0 & 77 \\
\hline Hebei & 71 & 52 & 31 & 36 & 70 & 62 & 53 & 36 & 51 \\
\hline Henan & 39 & 72 & 58 & 22 & 61 & 38 & 35 & 4 & 41 \\
\hline Anhui & 29 & 31 & 44 & 23 & 19 & 17 & 24 & 0 & 23 \\
\hline Tianjin & 19 & 12 & 9 & 12 & 22 & 20 & 17 & 12 & 15 \\
\hline Jilin & 15 & 9 & 6 & 15 & 21 & 17 & 19 & 3 & 13 \\
\hline Jiangsu & 25 & 16 & 3 & 22 & 0 & 4 & 9 & 5 & 11 \\
\hline Shandong & 15 & 14 & 11 & 12 & 13 & 12 & 10 & 4 & 11 \\
\hline Liaoning & 10 & 9 & 7 & 8 & 13 & 10 & 10 & 5 & 9 \\
\hline Shanxi & 4 & 7 & 4 & 4 & 8 & 6 & 5 & 3 & 5 \\
\hline Shaanxi & 2 & 4 & 2 & 2 & 4 & 3 & 2 & 1 & 3 \\
\hline Nei Mongol & 1 & 1 & 1 & 1 & 1 & 1 & 1 & 0 & 1 \\
\hline Shanghai & 3 & 1 & 0 & 2 & 0 & 0 & 1 & 0 & 1 \\
\hline Beijing & 1 & 1 & 0 & 0 & 1 & 1 & 1 & 1 & 1 \\
\hline Hubei & 0 & 3 & 2 & 0 & 2 & 1 & 0 & 0 & 1 \\
\hline Chongqing & 0 & 0 & 0 & 0 & 0 & 0 & 0 & 0 & 0 \\
\hline Hunan & 0 & 0 & 0 & 0 & 0 & 0 & 0 & 0 & 0 \\
\hline Sichuan & 0 & 1 & 0 & 0 & 1 & 1 & 0 & 0 & 0 \\
\hline Yunnan & 0 & 0 & 0 & 0 & 0 & 0 & 0 & 0 & 0 \\
\hline Zhejiang & 1 & 1 & 0 & 1 & 0 & 0 & 0 & 0 & 0 \\
\hline Guizhou & 0 & 0 & 0 & 0 & 0 & 0 & 0 & 0 & 0 \\
\hline Xinjiang Uygur & 0 & 0 & 1 & 0 & 1 & 0 & 0 & 0 & 0 \\
\hline Ningxia Hui & 0 & 1 & 0 & 1 & 1 & 1 & 0 & 0 & 0 \\
\hline Jiangxi & 0 & 0 & 0 & 0 & 0 & 0 & 0 & 0 & 0 \\
\hline Guangxi & 0 & 0 & 0 & 0 & 0 & 0 & 0 & 0 & 0 \\
\hline Fujian & 0 & 0 & 0 & 0 & 0 & 0 & 0 & 0 & 0 \\
\hline Gansu & 0 & 0 & 0 & 0 & 0 & 0 & 0 & 0 & 0 \\
\hline Guangdong & 0 & 0 & 0 & 0 & 0 & 0 & 0 & 0 & 0 \\
\hline Hainan & 0 & 0 & 0 & 0 & 0 & 0 & 0 & 0 & 0 \\
\hline Qinghai & 0 & 0 & 0 & 0 & 0 & 0 & 0 & 0 & 0 \\
\hline Xizang & 0 & 0 & 0 & 0 & 0 & 0 & 0 & 0 & 0 \\
\hline HongKong & 0 & 0 & 0 & 0 & 0 & 0 & 0 & 0 & 0 \\
\hline Macao & 0 & 0 & 0 & 0 & 0 & 0 & 0 & 0 & 0 \\
\hline Taiwan & 0 & 0 & 0 & 0 & 0 & 0 & 0 & 0 & 0 \\
\hline China & 338 & 319 & 263 & 237 & 334 & 277 & 280 & 374 & 263 \\
\hline
\end{tabular}



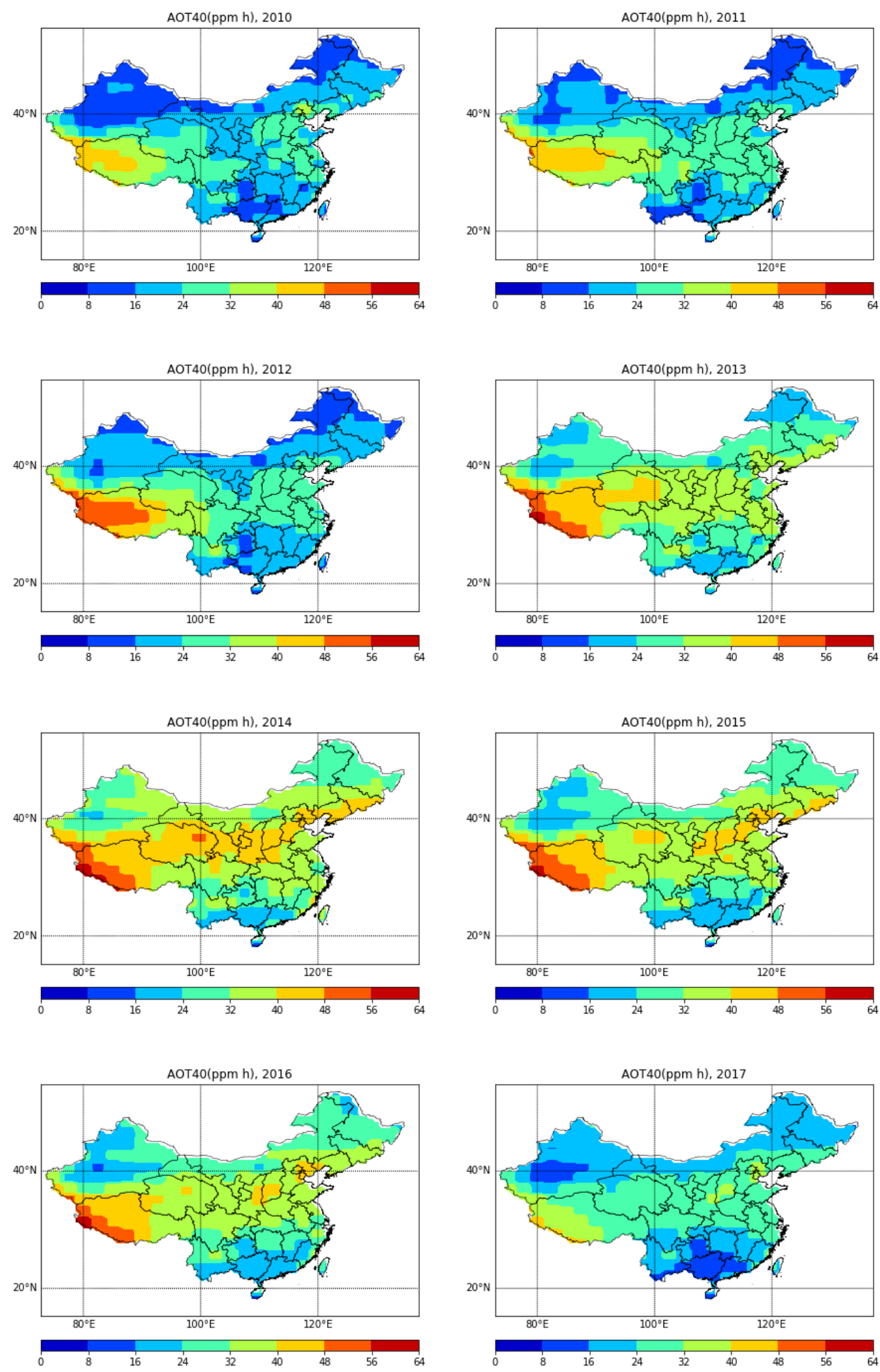

Fig. S1. The spatial distribution of the annual average accumulated AOT40 from 2010 to 2017 (unit of ppm h). 

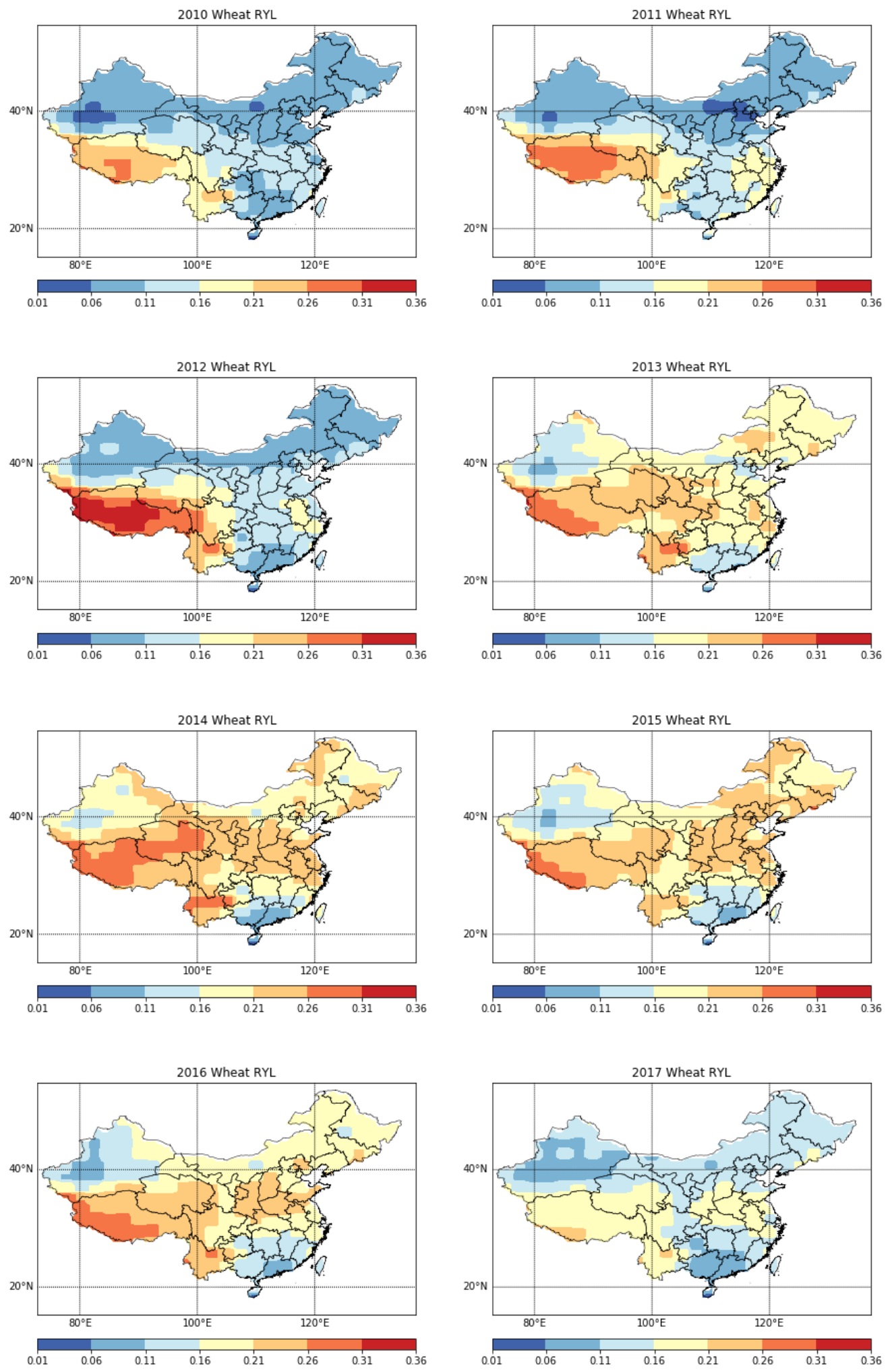

Fig. S2. Spatial distribution of relative yields loss (RYL) for wheat during growing season (March, April, May) from 2010 to 2017. 

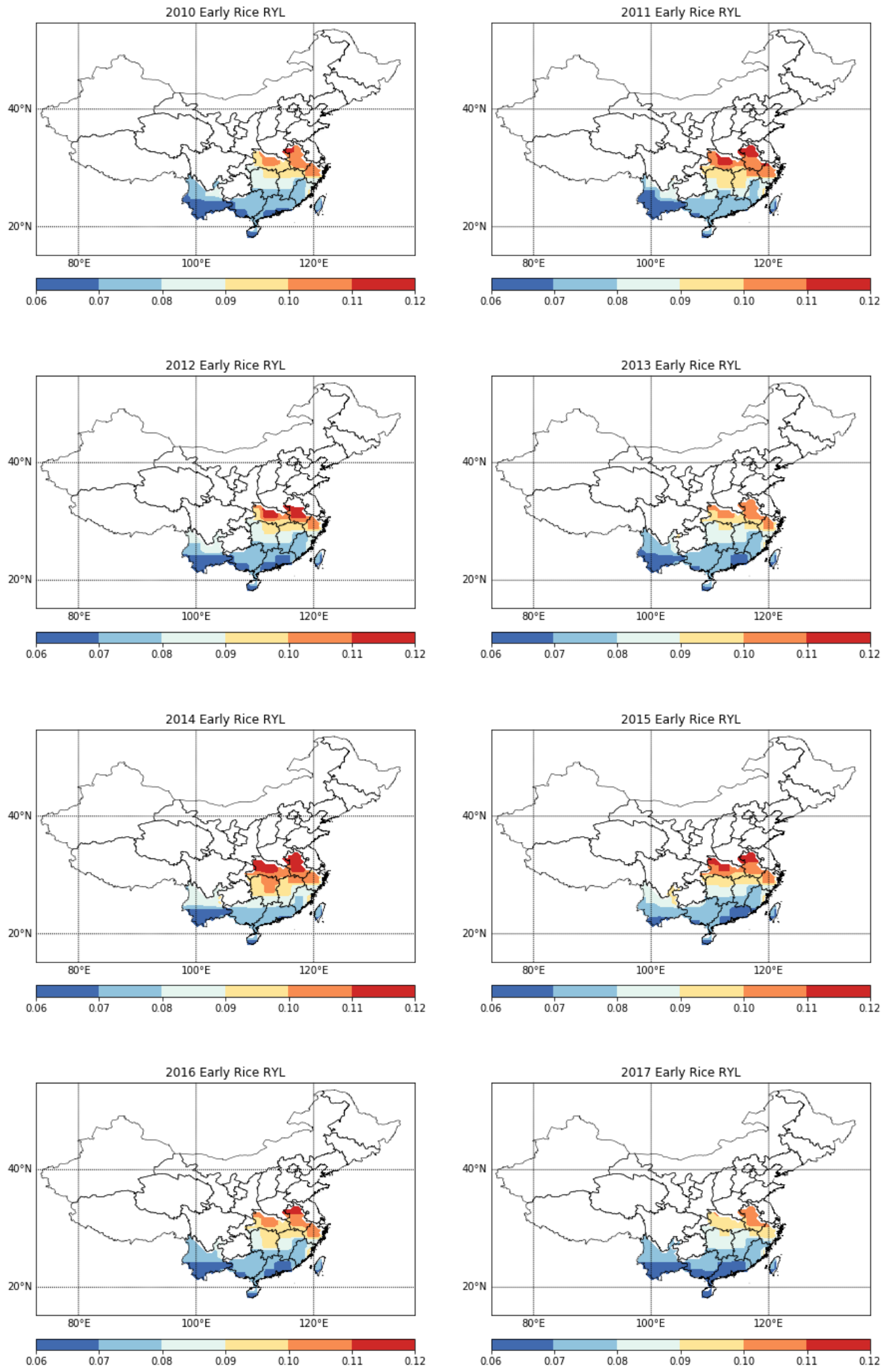

Fig. S3. As Fig. S2 but for double early rice (growing season May, June, July). 

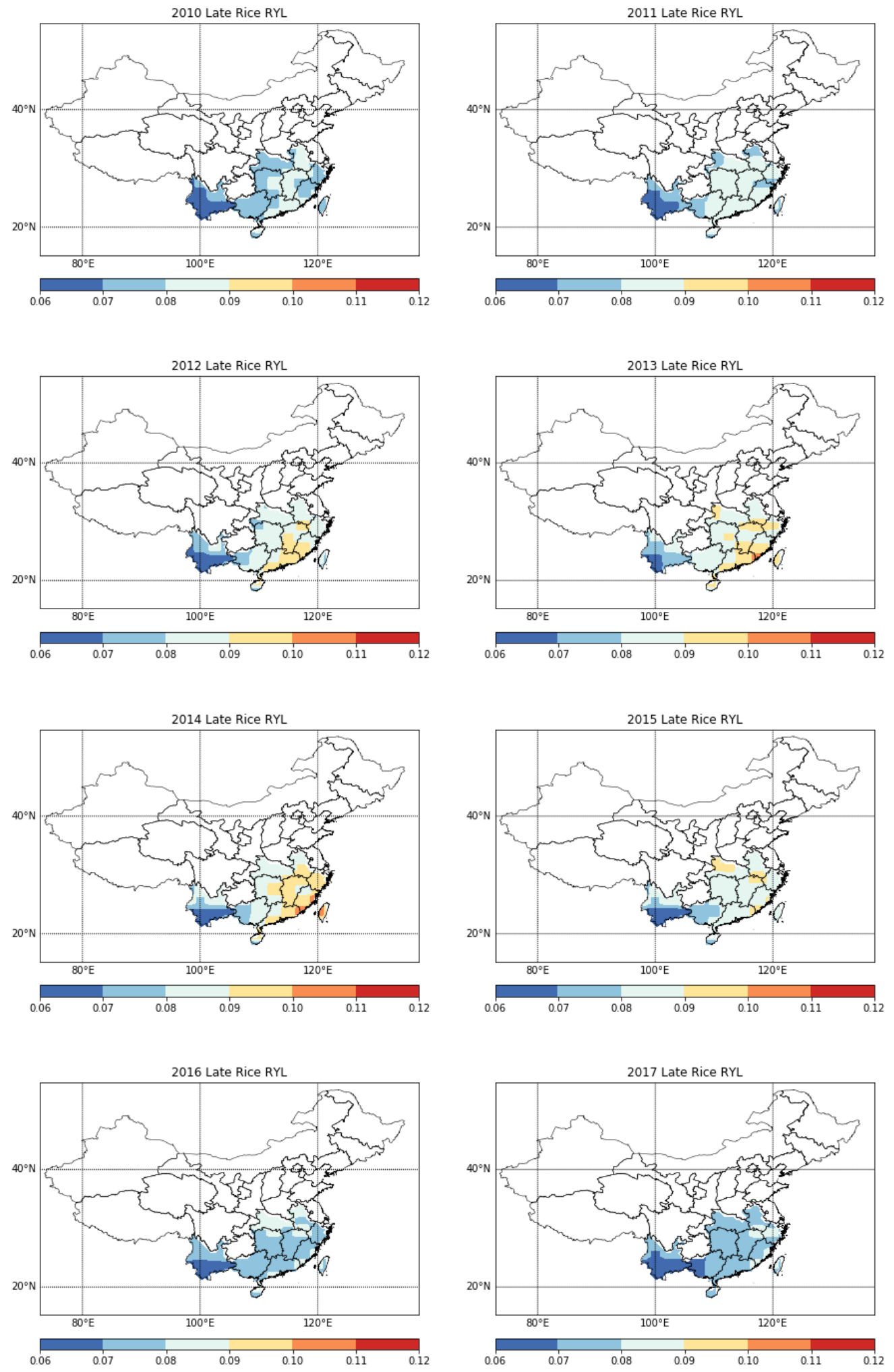

Fig. S4. As Fig. S2 but for double late rice (growing season September, October, November) 

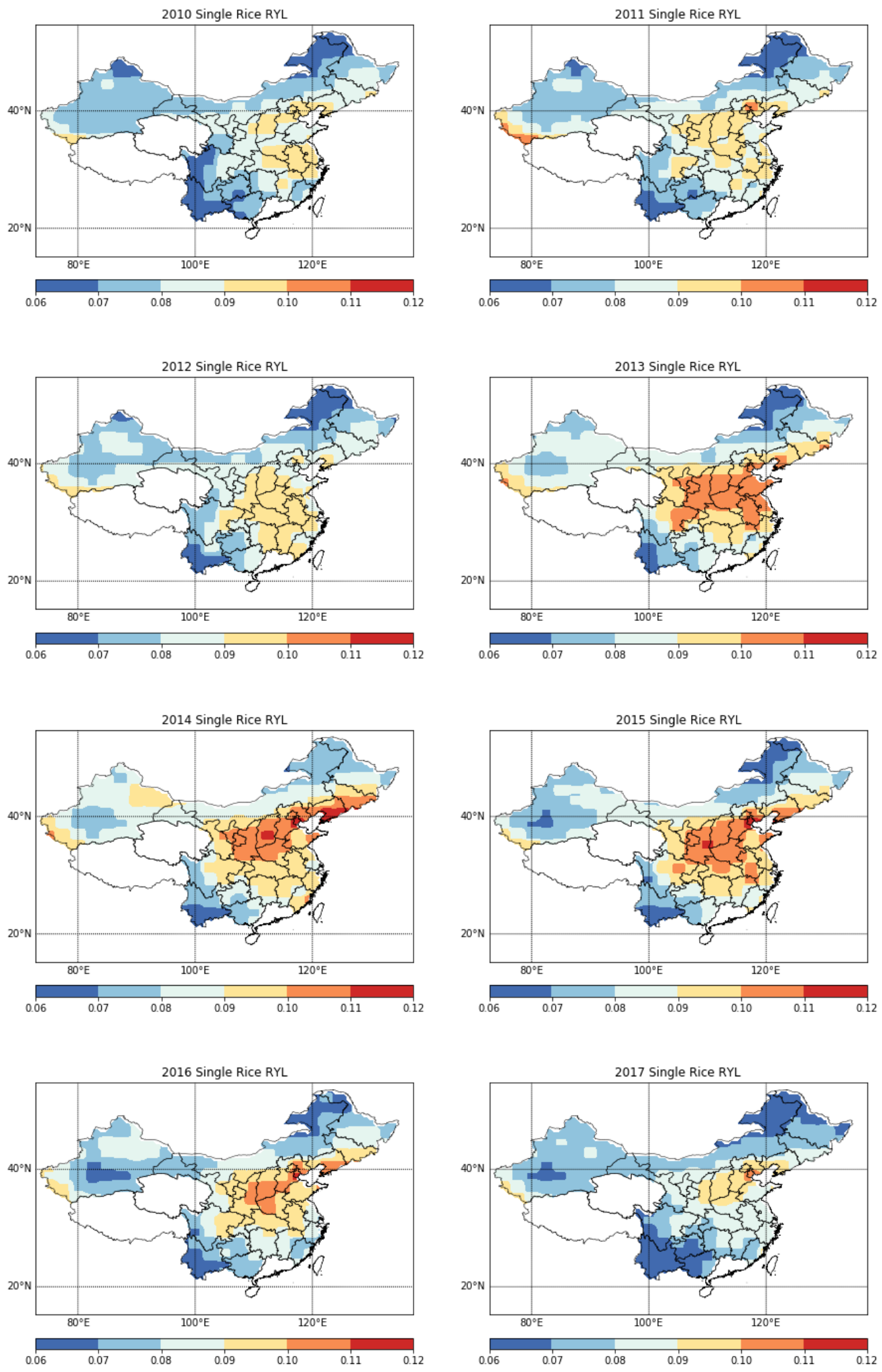

Fig. S5. As Fig. S2 but for single rice (growing season August, September, October). 

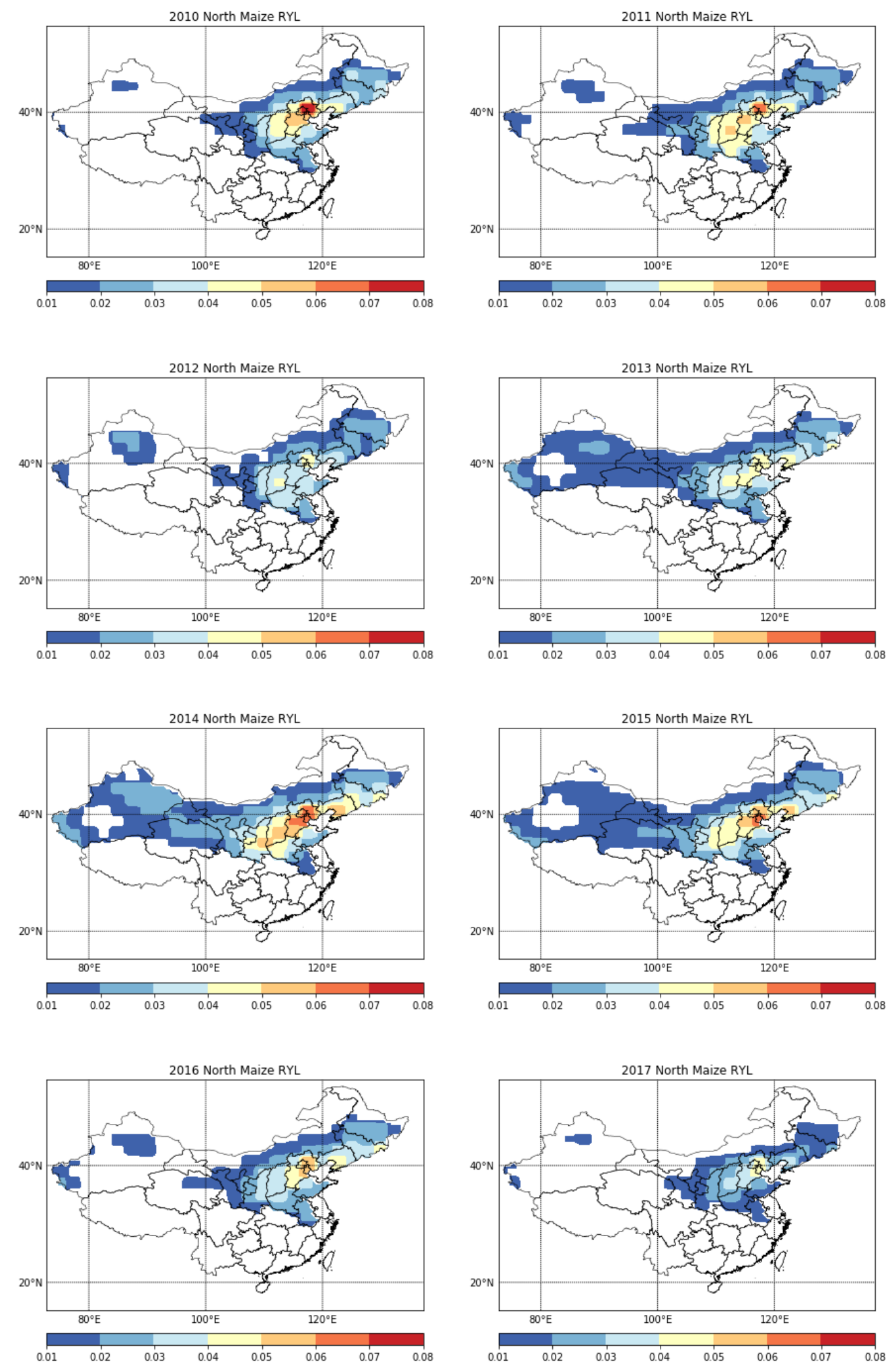

Fig. S6. As Fig. S2 but for north maize (growing season June, July, August). 

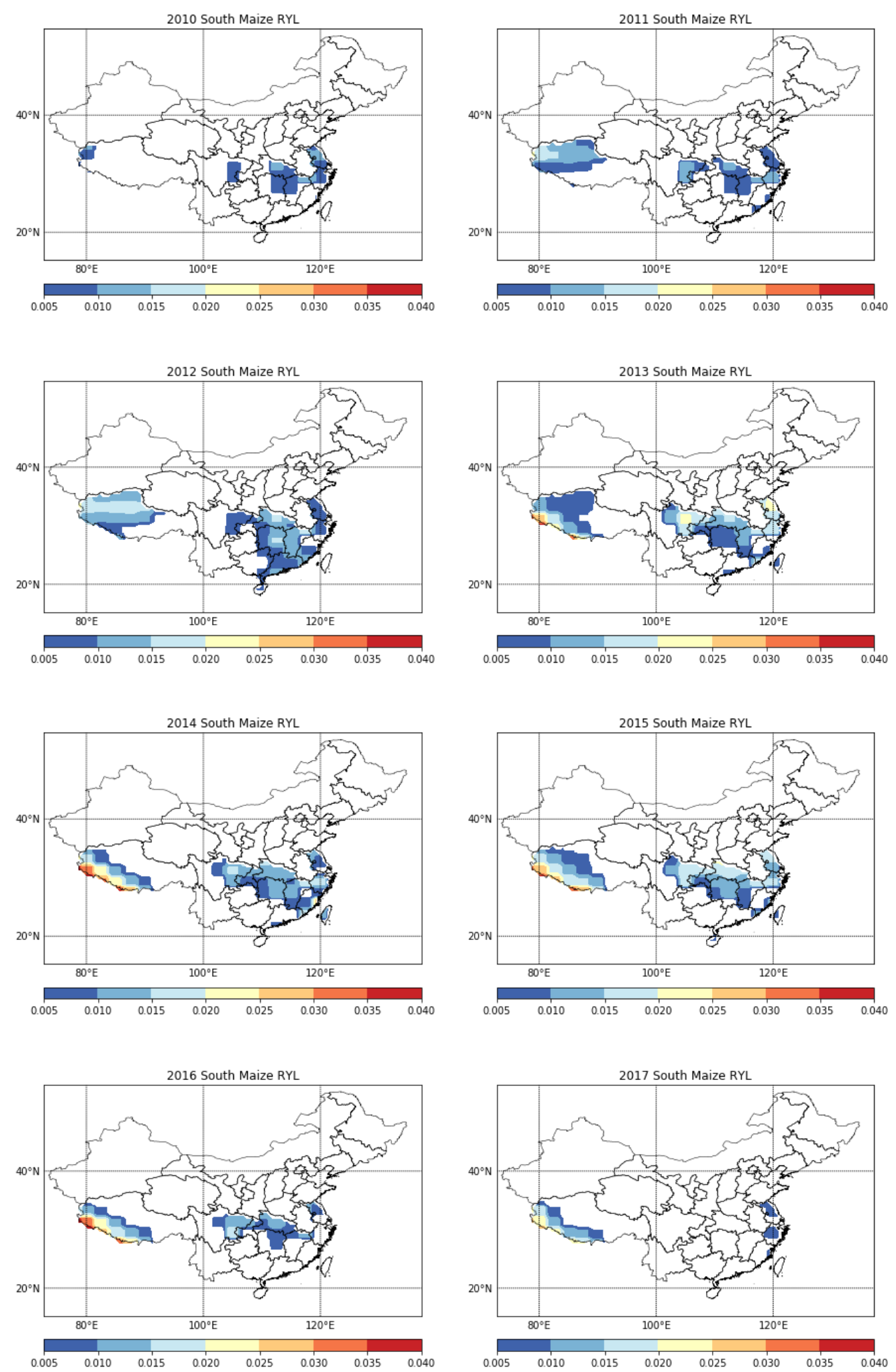

Fig. S7. As Fig. S2 but for south maize (growing season August, September, October). 

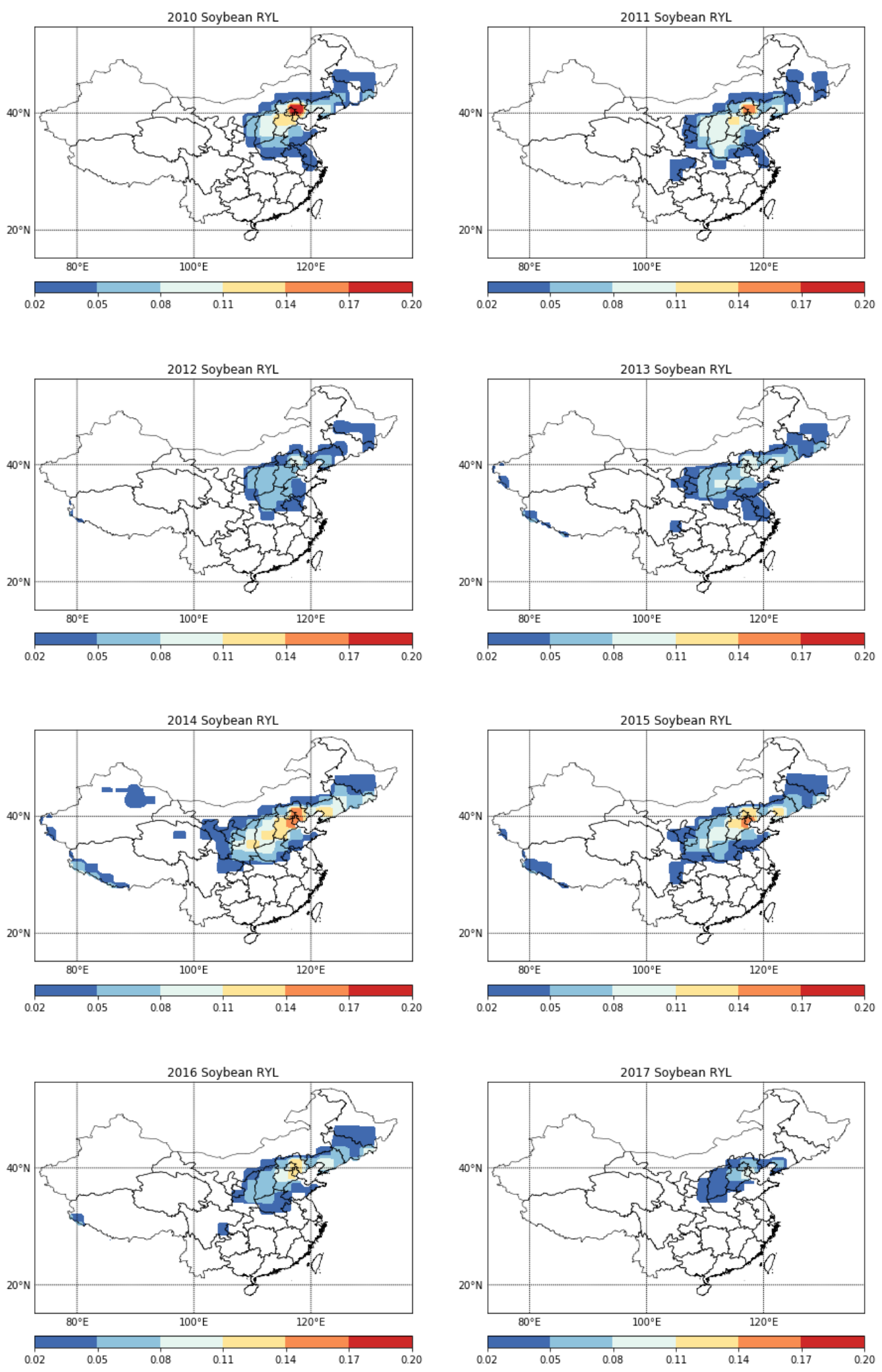

Fig. S8. As Fig. S2 but for soybean (growing season June, July, August). 
Fig. S9. Annual Maize Production loss by province from 2010 to 2017 (1000 Mt)
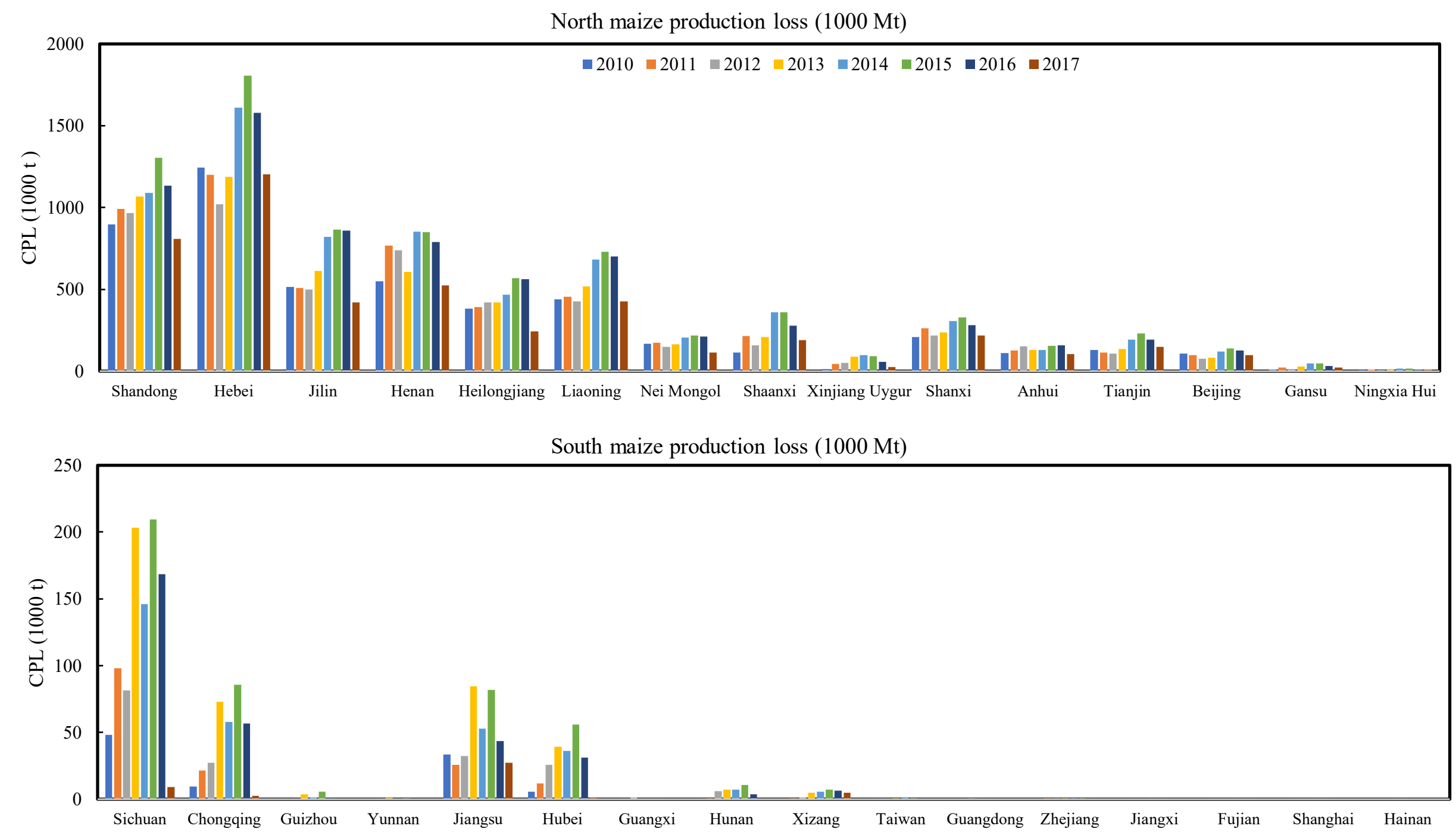
Fig. S10. Annual Soybean Production loss by province from 2010 to 2017 (1000 Mt)

Soybean Production Loss (1000 t)

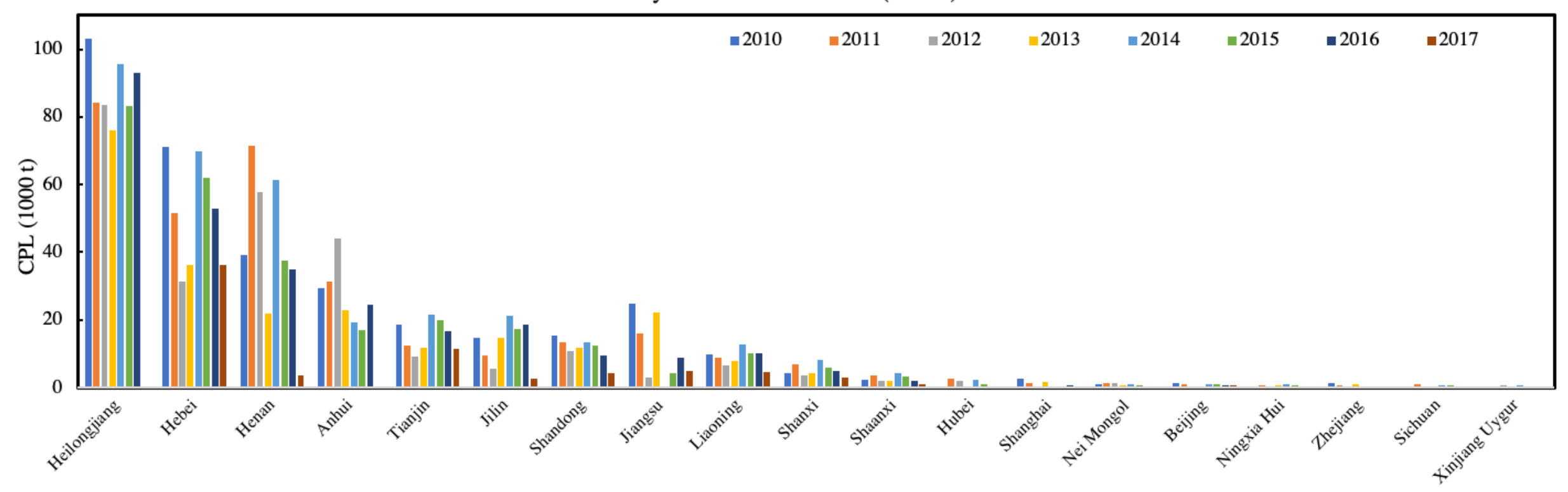

